\title{
Strong Reduction in Ge Film Reflectivity by an Overlayer of 3-nm Si Nanoparticles: Implications for Photovoltaics
}

Ayman Rezk ${ }^{1}$, Sabina Abdul Hadi ${ }^{2}$, Juveiriah M. Ashraf ${ }^{1}$, Aisha Alhammadi ${ }^{1}$, Wafa Alnaqbi ${ }^{1}$, Aravindh Kumar ${ }^{3}$, Ghada Dushaq $^{4}$, Mahmoud S Rasras ${ }^{4}$, Krishna C. Saraswat ${ }^{3}$, Munir Nayfeh ${ }^{5}$, and Ammar Nayfeh ${ }^{1, *}$

${ }^{1}$ Electrical Engineering and Computer Science, Khalifa University, Abu Dhabi, 127788, UAE.

${ }^{2}$ College of Engineering and IT, University of Dubai, Dubai, 14143, UAE.

${ }^{3}$ Electrical Engineering, Stanford University, Stanford, CA, 94305, USA.

${ }^{4}$ Electrical Engineering, New York University Abu Dhabi, Abu Dhabi, 129188, UAE.

${ }^{5}$ Department of Physics, University of Illinois, Champaign, IL, 61820, USA.

*email: ammar.nayfeh@ku.ac.ae

\section{This file includes:}

Figure S1. The reduction in R relative to uncoated samples for (a) PECVD Ge and (b) c-Si versus drop casted volume of Si NPs at various photon energies.

Table S1. Estimated Si NP thicknesses using Modified Transfer Matrix model with estimated optical properties of Si nanoparticle and PECVD Ge layer with AMF measured RMS surface roughness.

Figure S2. Calculated (a) Reflectance of Si NP/Si stack and (b) absorption in Si NP layers for increasing thickness of Si NP layer from 0-100 nm.

Figure S3. Estimated absorption for Si NP and absorption of c-Si for $10 \mathrm{~nm}$ and $100 \mathrm{~nm}$ thick layers. Absorption is calculated using Beer's law (1- $\left.e^{-\alpha x}\right)$ ignoring any reflectance $(R=0)$. 
(a)

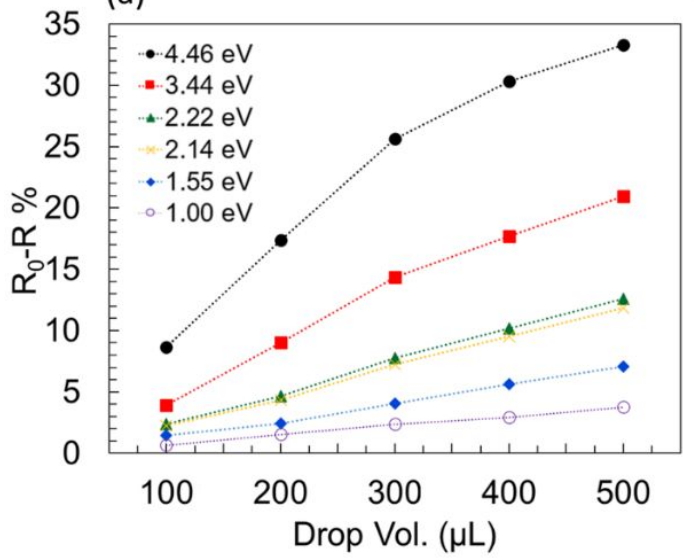

(b)

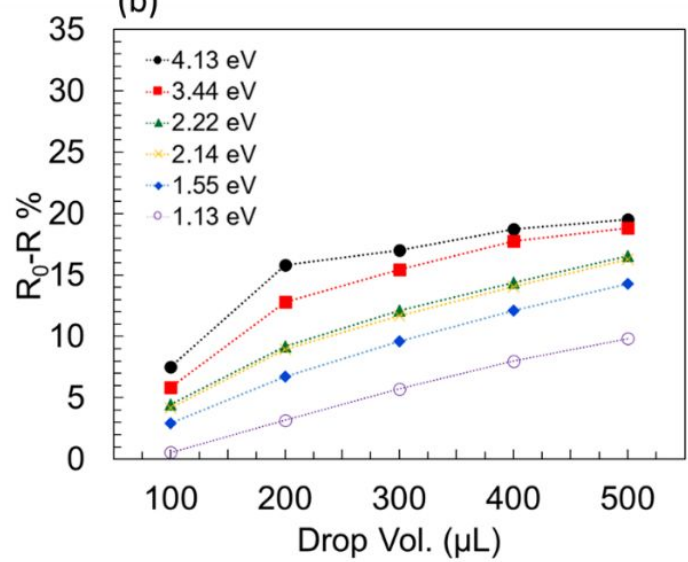

Fig. S1 | The reduction in R relative to uncoated samples for (a) PECVD Ge and (b) c-Si versus drop casted volume of Si NPs at various photon energies.

Table S1

Estimated Si NP thicknesses using Modified Transfer Matrix model with estimated optical properties of Si nanoparticle and PECVD Ge layer with AMF measured RMS surface roughness.

\begin{tabular}{|c|c|c|c|c|c|c|c|}
\hline Drop-volume & & $0 \mu \mathrm{L}$ & $100 \mu \mathrm{L}$ & $200 \mu \mathrm{L}$ & $300 \mu \mathrm{L}$ & $400 \mu \mathrm{L}$ & $500 \mu \mathrm{L}$ \\
\hline $\begin{array}{l}\text { MTM Model for } \\
\text { Si NP/ PECVD Ge/ }\end{array}$ & RMS Surface Roughness Rq (nm) & 2 & 4.5 & 6 & 6.5 & 7 & 8 \\
\hline $\begin{array}{l}\text { Si substrate stack } \\
\left(A F M R_{q}\right)\end{array}$ & Si NP estimated thickness ( $\mathrm{nm}$ ) & $10^{\mathrm{a}}$ & 18 & 23 & 28 & 32 & 35 \\
\hline Figure 9 (a) & RMSE for estimated-to-measured $R$ & 0.0199 & 0.0195 & 0.0173 & 0.0188 & 0.0212 & 0.0246 \\
\hline \multirow{2}{*}{$\begin{array}{l}\text { MTM Model for } \\
\text { Si NP/ PECVD Ge/ } \\
\text { Si substrate stack }\end{array}$} & RMS Surface Roughness Rq (nm) & 125 & 210 & 191 & 154 & 138 & 157 \\
\hline & Si NP estimated thickness (nm) & 0 & 0 & 16 & 26 & 30 & 34 \\
\hline \multicolumn{8}{|l|}{ ( $R_{q}$ as variable) } \\
\hline Figure 9 (b) & RMSE for estimated-to-measured R & 0.0173 & 0.0124 & 0.0131 & 0.0144 & 0.0161 & 0.0170 \\
\hline
\end{tabular}

${ }^{\text {a }}$ Model assumes $10 \mathrm{~nm}$ as opposed to $0 \mathrm{~nm}$, likely due to some level of errors in estimated optical data.

Wavelength $\lambda(\mathrm{nm})$

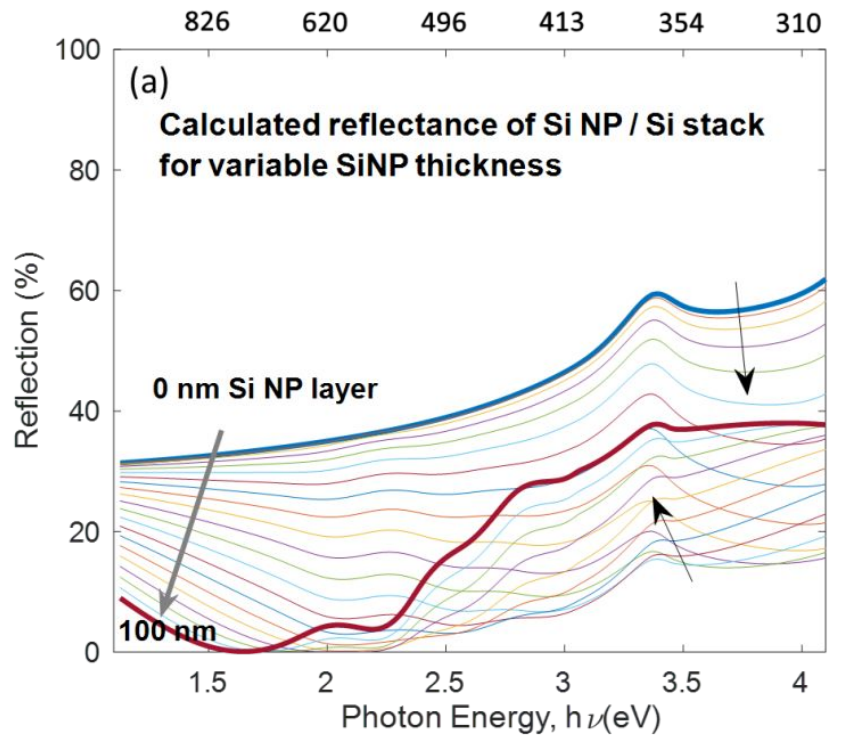

Wavelength $\lambda(\mathrm{nm})$

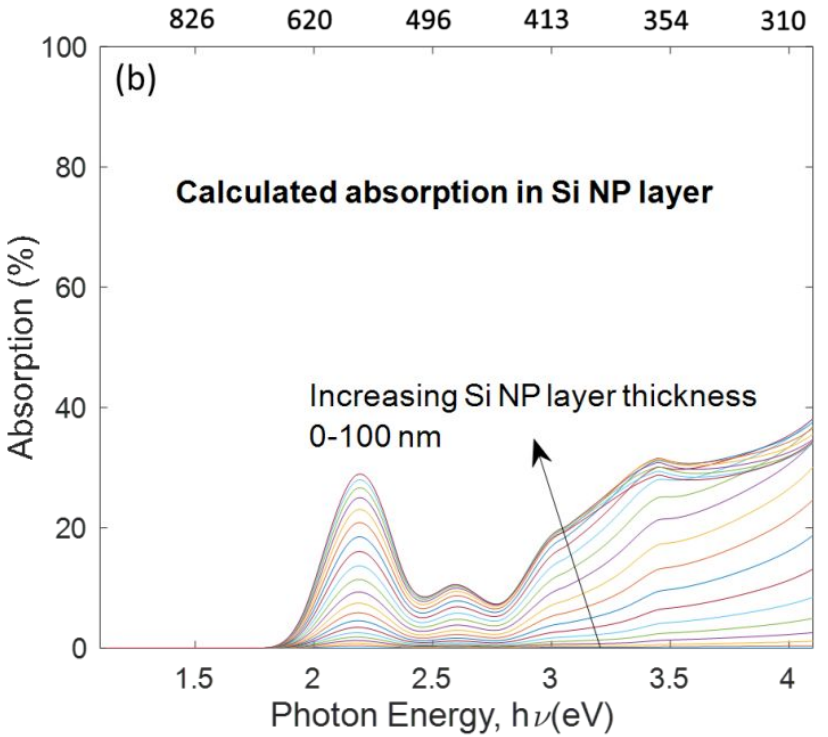

Fig. S2 | Calculated (a) Reflectance of Si NP/Si stack and (b) absorption in Si NP layers for increasing thickness of Si NP layer from 0-100 nm. 


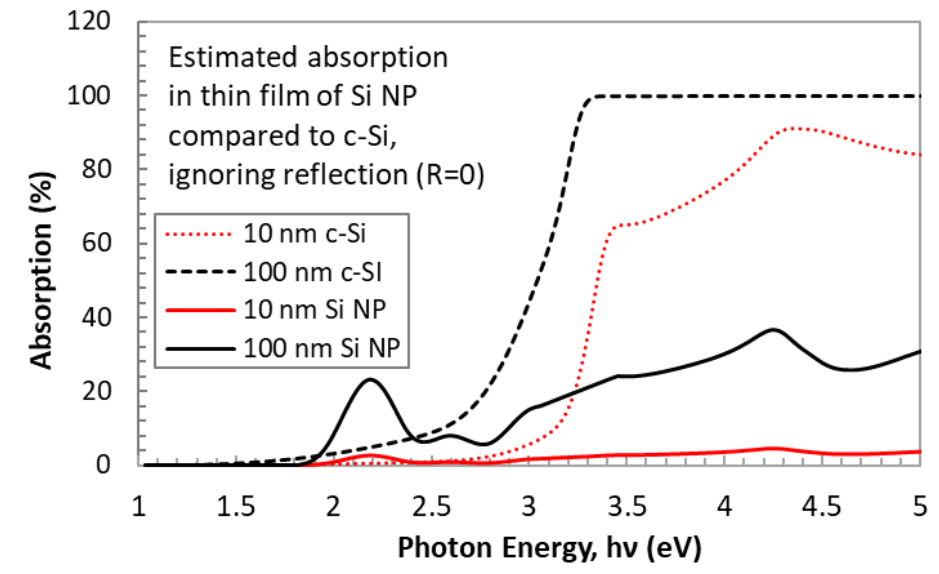

Fig. S3 | Estimated absorption for Si NP and absorption of c-Si for $10 \mathrm{~nm}$ and $100 \mathrm{~nm}$ thick layers. Absorption is calculated using Beer's law (1- $\mathrm{e}^{-\alpha x}$ ) ignoring any reflectance $(R=0)$. 